\title{
Propagación vegetativa de taique (Desfontainia spinosa) y tepa (Laureliopsis philippiana) con fines ornamentales
}

\author{
Vegetative propagation for horticultural potential of taique (Desfontainia spinosa) and \\ tepa (Laureliopsis philippiana) by cuttings
}

\author{
Mabel F Delgado ${ }^{a^{*}}$, Marely Cuba ${ }^{\text {b }}$ Paulina Hechenleitner ${ }^{\mathrm{a}}$, Oscar Thiers ${ }^{\mathrm{a}}$ \\ *Autor de correspondencia: aUniversidad Austral de Chile, Instituto de Silvicultura, casilla 567, Valdivia, Chile, \\ tel. 56-63-222296, mabeldelgado@uach.cl \\ bUniversidad de Concepción, Departamento de Ciencias Básicas, Los Ángeles, Chile.
}

\begin{abstract}
SUMMARY
Taique (Desfontainia spinosa) and tepa (Laureliopsis philippiana) are both highly ornamental native species from Chile. In order to investigate the capacity of vegetative propagation of these species, different concentrations of indolbuturic acid (IBA) with different types of cuttings were tested. The study was carried out inside a greenhouse with intermittent misting system. Cuttings were placed on a hot propagation bed at a temperature of $22 \pm 2^{\circ} \mathrm{C}$. A mixture of Sphagnum and perlite (1:1) was used as propagation substrate. IBA concentrations of 0-1,000-2,500 and 4,000 ppm were tested for apical and subapical cuttings of taique. Concentrations of $0,1,000,2,000,3,000$, and 4,000 ppm were tested for apical cuttings of tepa. Main results indicated that taique can be propagated easily by cuttings collected during winter time using IBA at concentrations between 1,000-4,000 ppm (67-82\% of rooting succeeded), no matter the type of cutting used. Tepa showed a greater difficulty in rooting capacity than taique when propagated by cuttings. Results showed values of propagation percentage from $6.7 \%$ for cuttings without hormone, up to $33 \%$ for cuttings with a concentration of 4,000 ppm of AIB.
\end{abstract}

Key words: Desfontainia spinosa, Laureliopsis philippiana, vegetative propagation, native foliages.

\section{RESUMEN}

Taique (Desfontainia spinosa) y tepa (Laureliopsis philippiana) son especies nativas de Chile con gran potencial ornamental. El objetivo de este estudio consistió en la propagación vegetativa de estas especies utilizando diferentes concentraciones de ácido indolbutírico (AIB) y tipos de esqueje. El ensayo se estableció dentro de un invernadero con sistema de riego nebulizado sobre cama de enraizamiento con temperatura controlada de $22 \pm 2^{\circ}$ C. Como sustrato de enraizamiento se utilizó una mezcla de Sphagnum y perlita (1:1). Para taique se utilizaron concentraciones de AIB de 0 (testigo), 1.000, 2.500 y $4.000 \mathrm{mg} \mathrm{L}^{-1}$ en esquejes apicales y subapicales. En tepa se probaron concentraciones de AIB de 0 (testigo), 1.000, 2.000, 3.000 y $4.000 \mathrm{mg} \mathrm{L}^{-1}$ en esquejes subapicales. Los resultados indicaron que taique se propagó fácilmente a través de esquejes colectados en invierno utilizando concentraciones de AIB de 1.000-4.000 $\mathrm{mg} \mathrm{L}^{-1}$ (67-82\%), sin importar el tipo de estaca utilizada. Por el contrario, tepa fue de difícil enraizamiento, cuyos valores oscilan entre $6,7 \%$ para los esquejes sin hormona y $33 \%$ para los esquejes tratados con $4.000 \mathrm{mg} \mathrm{L}^{-1}$ de AIB.

Palabras clave: Desfontainia spinosa, Laureliopsis philippiana, propagación vegetativa, follajes nativos.

\section{INTRODUCCIÓN}

La flora nativa del bosque templado húmedo valdiviano presenta numerosas especies de follaje siempreverde, que por sus particulares características de forma, tamaño, color y brillo son muy adecuadas y demandadas para uso ornamental (Tacón et al. 2005). Sin embargo, la variedad de especies nativas que actualmente se utiliza para este fin y que están disponibles en el mercado es mínima, comparada con la gran diversidad de flora existente en el país.

Actualmente especies nativas arbóreas como el avellano (Gevuina avellana (Molina)) y fuinque (Lomatia ferruginea (Cav.) R. Br.), y helechos como ampe (Lophosoria quadripinnata (J. F. Gmel.) C. Chr.), palma (Lycopodium paniculatum Desv.) y pon pon (Dendroligotrichum dendroides (Brid. ex Hedw) Broth) son recolectadas desde su hábitat natural, principalmente por familias campesinas e indígenas, y posteriormente se venden a florerías o a pequeñas empresas exportadoras de follaje para mercados en Estados Unidos, Japón, Canadá y Holanda, entre otros (Tacón et al. 2005).

Esta situación trae beneficios económicos y sociales; sin embargo, en Chile no existen referencias al manejo ni se ha investigado el impacto de la extracción de estas especies para asegurar que esta actividad se desarrolle de manera sustentable (Palma 2003). Además, muchas 
veces la extracción se realiza en forma indiscriminada, lo que conlleva a una falta de homogeneidad y calidad del producto.

Recientemente se han puesto en marcha proyectos productivos con apoyo estatal que intentan disminuir la presión de extracción sobre el bosque nativo (Tacón et al. 2005). Tal es el caso de la Asociación Indígena Mujeres Follajes San Juan, en la comuna de San Juan de la Costa, asesorada por el Instituto de Desarrollo Agropecuario (INDAP), y proyectos de la Fundación para la Innovación Agraria (FIA). Esta tarea recién comienza, ya que no se tienen antecedentes de cultivo comercial de las especies nativas que proporcionan follaje ornamental. Dicha tarea tiene su inicio en la selección de las plantas que presentan características ornamentales idóneas para el mercado, para luego buscar la forma de propagar vegetativamente estas especies y a largo plazo obtener plantas que presenten homogeneidad en la calidad del follaje y permitan mantener un flujo de abastecimiento y una disponibilidad continua para la comercialización de éstas (FIA 2005).

El presente trabajo tiene por objetivo general evaluar la propagación vegetativa con fines ornamentales de las especies taique (Desfontainia spinosa (Ruiz et Pav.)) y tepa (Laureliopsis philippiana (Looser) Schodde), por poseer ellas un gran potencial para ser exportadas debido a sus características de flores y follaje, respectivamente. Taique tiene una inflorescencia uniflora, tubuliforme y axilar, de 3 a $5 \mathrm{~cm}$ de largo, de color anaranjado a rojo, con lóbulos amarillos en los extremos, presentando un hermoso contraste con el color del follaje verde oscuro brillante (Donoso y Cabello 1978). Tepa es ornamental esencialmente por su follaje verde lustroso y perfumado (Riedemann y Aldunate 2003). Además, ambas especies presentan una elegante forma de sus hojas y una larga vida postcosecha (> 10 días), lo que las favorece notablemente para la exportación ${ }^{1}$.

Taique es un arbusto siempreverde que se distribuye ampliamente entre las provincias de Linares y Magallanes, entre ambas cordilleras sobre 600 m s.n.m. Habita lugares muy húmedos y soporta bajas temperaturas. Ocupa parte del sotobosque en bosques de alerce (Fitzroya cupressoides (Mol.) Johnston.), araucaria (Araucaria araucana (Mol.) K. Koch.) y coihue (Nothofagus dombeyi (Mirb.) Oerst.) (Donoso 1983). Se propaga por semilla en almácigo estratificado en otoño, en una mezcla de suelo de una parte de compost, una de tierra ácida y media de arena, repicándose a bolsa con la misma mezcla cuando tiene aproximadamente $1 \mathrm{~cm}$ de altura (Riedemann y Aldunate 2003). Su reproducción vegetativa presenta altos porcentajes de enraizamiento (69 y $84 \%$ ) con o sin el uso de hormona de enraizamiento en estacas colectadas en época primavera-verano (Awad 1993). Sin embargo, Riedemann y Aldunate (2003) indican que tardan bastante en enraizar (ocho meses). Florece durante

1 Antonio Ceballos, empresa Southgreen, comunicación personal. enero-febrero. Adecuada en una posición de semisombra y protegida, pero de difícil aclimatación fuera de su zona de distribución. No apta para suelos alcalinos y arcillosos (Donoso 1983).

Tepa crece sólo en Chile y escasamente en Argentina. En Chile se encuentra desde las provincias de Arauco y Bío-Bío (37³0' S) hasta el sur de Aysén en los $47^{\circ} 30^{\prime} \mathrm{S}$ (Donoso 2006). Se desarrolla favorablemente en suelos húmedos, profundos, ricos en nutrientes y de $\mathrm{pH}$ ácido (Donoso 1983). Su reproducción por semillas generalmente es baja, ya que altos porcentajes de éstas son inviables. Además, presenta ciertos problemas para su reproducción vegetativa por medio de estacas (Donoso 2006). En un trabajo $^{2}$ donde se utilizaron concentraciones de AIB de $500,1.000$ y $2.000 \mathrm{mg} \mathrm{L}^{-1}$, más los esquejes testigos, se obtuvieron resultados de enraizamiento de $33 \%$ con la concentración más alta utilizada $\left(2.000 \mathrm{mg} \mathrm{L}^{-1}\right)$ a los 10 meses de establecido el ensayo en cama fría con sustrato de Sphagnum y arena (1:1). Por otro lado, Santelices (1990) constató que tepa se puede reproducir vegetativamente a partir de estacas colectadas en otoño con una concentración de $1.000 \mathrm{mg} \mathrm{L}^{-1}$ de AIB y con niveles óptimos de temperatura para la producción de raíces (número y longitud) y supervivencia (97\%) de 20 y $25^{\circ} \mathrm{C}$ a los 10 meses de instalado el ensayo. Además probó distintas distancias de corte al ápice $(0-15,15-30,>30 \mathrm{~cm})$, obteniendo los mejores resultados de enraizamiento aquellas estacas extraídas a una distancia mayor a $15 \mathrm{~cm}$ del ápice, es decir, entre $15-30 \mathrm{y}>30 \mathrm{~cm}$.

Teniendo en cuenta estos antecedentes se planteó como objetivo específico determinar los porcentajes de supervivencia y de enraizamiento y el efecto de la aplicación de distintas concentraciones de AIB en el desarrollo del sistema radical y aéreo de taique y tepa. En el caso de taique, innovando distintos tipos de estaca de tallo y en el caso de tepa probando un mayor rango de concentraciones de AIB (0-4.000 mg L $\left.\mathrm{m}^{-1}\right)$ para obtener un mayor control del efecto del ácido con respecto al enraizamiento.

\section{MÉTODOS}

El ensayo se realizó en un invernadero rústico tipo túnel con cubierta de polietileno perteneciente a la Empresa Southgreen, dedicada a la recolección y exportación de follajes nativos. Este se encuentra ubicado en la provincia de Llanquihue, Ruta 5 sur, a $5 \mathrm{~km}$ de la ciudad de Puerto Varas, Región de Los Lagos. El período de estudio fue de cinco meses (julio-noviembre de 2006).

Para el enraizamiento de los esquejes se utilizó una cama de propagación caliente, cuya temperatura fue regulada por un termostato a $22 \pm 2{ }^{\circ} \mathrm{C}$. El sistema de riego fue del tipo nebulizador activado manualmente tres veces al

2 Bernardo Escobar, Universidad Austral de Chile, datos no publicados, comunicación personal. 
día (9:00, 13:00 y 17:00 horas), con una duración de cada riego de un minuto. Posteriormente, debido al aumento de las temperaturas producto del cambio estacional de invierno a primavera, el riego fue ampliado a un mayor número de aplicaciones. Este fluctuó de tres veces diarias cuando la temperatura no excedía los $20^{\circ} \mathrm{C}$ hasta seis o más veces cuando sobrepasaba este valor, pero con tiempos de riego de menor duración (30 segundos). El sustrato de propagación consistió en una mezcla de Sphagnum $s p$. y perlita en partes iguales.

El material vegetativo se colectó de su ambiente natural los primeros días de julio de 2006, en la Región de Los Lagos. Éste se seleccionó de la sección media de la planta, tomando en cuenta las características fenotípicas requeridas para follaje ornamental de exportación especificadas por la empresa ${ }^{3}$. La colecta se realizó de plantas madres visualmente sanas, es decir, sin presencia de hojas cloróticas que indicasen enfermedades o deficiencias nutricionales. Una vez seleccionadas las plantas, el material vegetal fue cortado con tijeras podadoras limpias y colocado dentro de cajas de poliestireno expandido con Sphagnum sp. en su interior para conservar la humedad ${ }^{4}$. Finalmente fueron transportadas a invernadero al día siguiente de su extracción, donde se procedió a la preparación de las estacas. Ésta consistió en la selección de aquellas estacas rectas, con buen vigor y sanidad, las que fueron cortadas aproximadamente de 6 a $10 \mathrm{~cm}$ de longitud, con un par de hojas. Estas últimas se redujeron a la mitad para evitar pérdidas por deshidratación en el invernadero. Se realizó una lesión (herida) longitudinal en la epidermis de la base de la estaca, de aproximadamente $2 \mathrm{~cm}$, con el fin de aumentar la superficie de absorción de ácido y así fomentar la producción de raíces.

Cada estaca fue expuesta por cinco segundos en una solución auxínica (AIB) en distintas concentraciones según la especie y finalmente montada sobre el sustrato de enraizamiento a unos 3-4 $\mathrm{cm}$ de profundidad y a una distancia de $10 \mathrm{~cm}$, presionando el sustrato alrededor de la estaca para lograr una buena superficie de contacto.

El diseño experimental para taique contempló dos factores: tipo de estaca y concentración de hormona. Los tipos de estaca fueron apicales (correspondiente a madera semidura, colectada hasta los $15 \mathrm{~cm}$ a partir del ápice) y subapicales (referidas a madera del año anterior, sacadas de la sección intermedia de la rama, es decir, de los 15 a 30 $\mathrm{cm}$ del ápice) y las concentraciones de hormona utilizadas fueron 0 (testigo) - $1.000-2.500$ y $4.000 \mathrm{mg} \mathrm{L}^{-1}$ de AIB. La combinación de éstos dio origen a ocho tratamientos, formados por 10 estacas y repetidos tres veces cada uno en

3 Empresa Southgreen.

4 Escobar B, F Utreras. Taller de propagación vegetativa por estacas. Proyecto Fondef D0111155: Desarrollo de tecnologías para la generación de una oferta diversificada de especies leñosas para el manejo de los bosques sustentables de Chile. Valdivia, 23 de abril de 2003. forma completamente al azar. En tepa se planteó un diseño estadístico completamente al azar, con cinco tratamientos (testigo, 1.000, 2.000, 3.000 y $4.000 \mathrm{mg} \mathrm{L}^{-1}$ de AIB), tres repeticiones y 10 estacas por tratamiento.

Luego de transcurridos tres meses en el caso de taique y cuatro meses en tepa se evaluaron: porcentajes de supervivencia y de enraizamiento, grado de enraizamiento (según la escala: $0=$ estaca sin callo, $1=$ estaca con callo, 2 = estaca con raíces menores a $1 \mathrm{~cm}, 3=$ estacas con raíces entre $1-3 \mathrm{~cm}, 4=$ estacas con raíces mayores a 3 $\mathrm{cm}$ ), largo de la raíz principal, número de raíces, largo de brotes, número de brotes, grado desarrollo de brotes (según la escala: $0=$ esquejes sin brotes, $1=$ brotes sin hoja abierta, 2 = brotes desarrollados con una hoja semiabierta, es decir, hoja débil y enroscada, $3=$ brotes desarrollados con una hoja abierta, o sea, vigorosa y bien formada, 4 = brotes desarrollados con dos hojas abiertas) y número de hojas viejas.

Para evaluar las diferencias en el tipo de estaca y las distintas concentraciones utilizadas en taique se realizó un análisis factorial (dos tipos de estaca $\mathrm{x}$ cuatro concentraciones). Se utilizó la prueba no paramétrica de Kruskal Wallis (con $P \leq 0,05$ ). Para comparar las distintas concentraciones de AIB en el desarrollo radical y aéreo de ambas especies se realizó análisis de varianza simple y la prueba de comparación múltiple de medias LSD, con un nivel de confianza de un $95 \%$.

\section{RESULTADOS}

Tipo de estaca de tallo para taique. No se encontraron diferencias significativas $(P>0,05)$ entre ambos tipos de estaca en todas las variables evaluadas (grado enraizamiento, número de raíces, longitud raíz principal, largo brotes, número de brotes, grado desarrollo brotes y número de hojas viejas) y las distintas concentraciones utilizadas $\left(0,1000,2.000\right.$ y $4.000 \mathrm{mg} \mathrm{L}^{-1}$ de AIB). Ello pudo estar influido por la escasa diferencia de lignificación de las estacas apicales y subapicales.

Porcentaje de supervivencia y enraizamiento de taique y tepa. Taique presentó un alto porcentaje de supervivencia ( $93 \%$ en promedio) para los tratamientos que utilizaron hormona. Sin embargo, en el tratamiento testigo se observó una menor supervivencia (77\%), como consecuencia del menor enraizamiento (figura 1A). Además se observó que en los esquejes con hormona el porcentaje de enraizamiento fue superior a los esquejes testigos $(38 \%, 67 \%, 77 \%$ y $82 \%$ para los tratamientos testigo, $1.000,2.500$ y $4.000 \mathrm{mg} \mathrm{L}^{-1}$, respectivamente). Por ello se enfatiza la importancia de la hormona AIB en la supervivencia y formación de raíces adventicias, ya que la mayoría de los esquejes a los cuales se les aplicó lograron permanecer activos y formaron un sistema radical. 


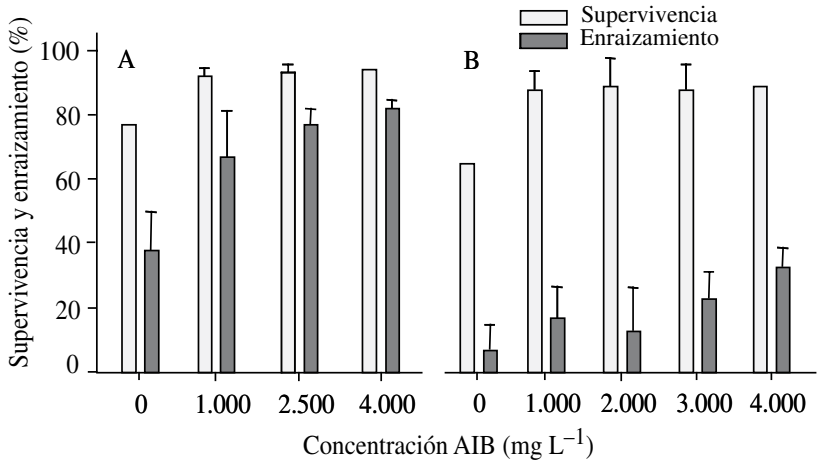

Figura 1. Porcentaje de supervivencia y enraizamiento de taique (A) y tepa (B) en función de las distintas concentraciones de AIB.

Taique (D. spinosa) (A) and tepa (L. philippiana) (B) survival and rooting percentage at different IBA concentrations.

Tepa logró un alto porcentaje de supervivencia (85\% en promedio) transcurridos los 120 días desde su plantación en el ensayo; sin embargo, su porcentaje de enraizamiento fue muy bajo, de sólo $7,17,13,23$ y $33 \%$ para los tratamientos testigo, $1.000,2.000,3.000$ y $4.000 \mathrm{mg} \mathrm{L}^{-1}$, respectivamente, siendo esta última concentración la más favorable para el enraizamiento (figura 1B).

Sistema radical y aéreo de taique. Todas las variables evaluadas, con la excepción del número de hojas viejas, presentaron un aumento en sus valores medios a medida que aumentó la concentración de AIB (cuadro 1). Por otro lado, se observó una diferencia significativa entre el testigo y los tratamientos con AIB. Además, en todas las variables evaluadas las concentraciones 2.500 y 4.000 $\mathrm{mg} \mathrm{L}^{-1}$ de AIB no provocaron diferencias significativas entre ellas.

Por otra parte, cabe destacar que las desviaciones estándar presentaron valores altos a muy altos, debido a que hubo esquejes que desarrollaron un sistema aéreo y radical bastante adecuado, mientras otras no lograron formar o elongar el primordio radical ni desarrollaron brotes.

Sistema radical y aéreo de tepa. La producción media de brotes pasados 120 días desde el establecimiento del ensayo mostró que la generalidad de los esquejes perdió sus hojas o mantuvo una, presentando al menos un brote desarrollado con una hoja semiabierta (cuadro 1).

En cuanto al desarrollo radicular (grado de enraizamiento, longitud y número de raíces) se observaron diferencias significativas a medida que aumentó la concentración de hormona, destacándose que la dosis más alta utilizada de ésta favoreció el desarrollo de las raíces de los esquejes. Así, por ejemplo, los esquejes tratados con $4.000 \mathrm{mg} \mathrm{L}^{-1}$ presentaron en promedio raíces principales de $1,2 \mathrm{~cm}$ de largo y un total de 1,9 raíces, lo cual difiere significativamente del resto de los tratamientos, en donde la mayoría de los esquejes sólo logró formar callo en su base.

Además, al igual que en taique, las desviaciones estándar fueron altas a muy altas.

Cuadro 1. Producción media* $( \pm S)$ de raíces y brotes de taique y tepa en función de los distintos tratamientos con AIB**. Average production* of taique (D. spinosa) and tepa (L. philippiana) roots and buds at different IBA treatments.

\begin{tabular}{|c|c|c|c|c|c|c|c|}
\hline \multirow{2}{*}{$\begin{array}{l}\text { Tratamiento } \\
\qquad \text { AIB } \\
\left(\mathrm{mg} \mathrm{L}^{-1}\right)\end{array}$} & \multicolumn{3}{|c|}{ Desarrollo raíces } & \multicolumn{4}{|c|}{ Desarrollo aéreo } \\
\hline & $\begin{array}{c}\text { Grado de } \\
\text { enraizamiento }\end{array}$ & $\begin{array}{l}\text { Longitud } \\
\text { de raíz } \\
(\mathrm{cm})\end{array}$ & $\begin{array}{l}\mathrm{N}^{\circ} \text { de } \\
\text { raíces }\end{array}$ & $\begin{array}{l}\text { Largo de } \\
\text { brotes } \\
(\mathrm{cm})\end{array}$ & $\begin{array}{l}\mathrm{N}^{\mathrm{o}} \text { de } \\
\text { brotes }\end{array}$ & $\begin{array}{l}\text { Grado de } \\
\text { desarrollo } \\
\text { de brotes }\end{array}$ & $\begin{array}{c}\mathrm{N}^{\circ} \text { de hojas } \\
\text { viejas }\end{array}$ \\
\hline \multicolumn{8}{|c|}{ Taique } \\
\hline 0 & $1,4 \pm 1,3 b$ & $0,8 \pm 2,7 \mathrm{c}$ & $1,8 \pm 1,2 \mathrm{c}$ & $0,1 \pm 0,3 \mathrm{~b}$ & $0,2 \pm 0,5 b$ & $0,3 \pm 0,8 \mathrm{~b}$ & $2,0 \pm 0,7 \mathrm{a}$ \\
\hline 1.000 & $2,6 \pm 2,2 \mathrm{a}$ & $2,7 \pm 3,1 \mathrm{~b}$ & $3,7 \pm 3,5 b$ & $0,3 \pm 0,4 \mathrm{a}$ & $0,3 \pm 0,3 b$ & $0,5 \pm 0,8 \mathrm{a}$ & $1,9 \pm 0,6 \mathrm{a}$ \\
\hline 2.500 & $2,7 \pm 1,5 \mathrm{a}$ & $3,2 \pm 3,5 \mathrm{a}$ & $5,4 \pm 2,6 \mathrm{a}$ & $0,3 \pm 0,3 \mathrm{a}$ & $0,6 \pm 0,7 \mathrm{a}$ & $0,5 \pm 0,8 \mathrm{a}$ & $2,0 \pm 0,4 \mathrm{a}$ \\
\hline 4.000 & $3,2 \pm 1,4 \mathrm{a}$ & $3,5 \pm 4,8 \mathrm{a}$ & $6,0 \pm 2,7 \mathrm{a}$ & $0,3 \pm 0,5 \mathrm{a}$ & $0,7 \pm 0,7 \mathrm{a}$ & $0,6 \pm 0,5 \mathrm{a}$ & $2,0 \pm 0,5 \mathrm{a}$ \\
\hline \multicolumn{8}{|c|}{ Tepa } \\
\hline 0 & $1,0 \pm 0,8 \mathrm{c}$ & $0,2 \pm 0,1 \mathrm{c}$ & $0,2 \pm 0,2 \mathrm{c}$ & $0,6 \pm 0,9 \mathrm{c}$ & $1,4 \pm 0,9 b$ & $2,2 \pm 1,4 \mathrm{a}$ & $0,5 \pm 0,8 \mathrm{a}$ \\
\hline 1.000 & $1,4 \pm 0,9 b$ & $0,5 \pm 0,2 \mathrm{c}$ & $0,4 \pm 0,4 \mathrm{c}$ & $0,9 \pm 0,9 \mathrm{c}$ & $1,4 \pm 0,9 b$ & $2,1 \pm 1,5 \mathrm{a}$ & $0,8 \pm 0,8 \mathrm{a}$ \\
\hline 2.000 & $1,2 \pm 0,9 b$ & $0,2 \pm 0,2 \mathrm{c}$ & $0,2 \pm 0,4 \mathrm{c}$ & $0,8 \pm 0,9 \mathrm{c}$ & $1,3 \pm 0,9 b$ & $1,8 \pm 1,4 \mathrm{a}$ & $0,8 \pm 0,8 \mathrm{a}$ \\
\hline 3.000 & $1,3 \pm 1,0 \mathrm{~b}$ & $0,7 \pm 0,5 b$ & $0,7 \pm 0,7 \mathrm{~b}$ & $1,0 \pm 1,1 \mathrm{~b}$ & $1,2 \pm 0,9 b$ & $2,1 \pm 1,4 \mathrm{a}$ & $0,7 \pm 0,8 \mathrm{a}$ \\
\hline 4.000 & $1,7 \pm 1,1 \mathrm{a}$ & $1,2 \pm 0,6 \mathrm{a}$ & $1,9 \pm 1,8 \mathrm{a}$ & $1,5 \pm 1,1 \mathrm{a}$ & $1,6 \pm 1,0 \mathrm{a}$ & $2,3 \pm 1,4 \mathrm{a}$ & $0,8 \pm 0,8 \mathrm{a}$ \\
\hline
\end{tabular}

* Valores medios con letras distintas indican diferencias significativas a un nivel de confianza de un 95\%. Different letters indicate average values with significant differences at $95 \%$ confidence level.

** Raíces: luego de 95 días; aéreo: luego de 120 días de iniciado el ensayo. Roots: after 95 days; aerial: after 120 days. 


\section{DISCUSIÓN}

La época de colecta fue realizada en pleno invierno, estación correspondiente al período de reposo vegetativo de las plantas, lo que pudiera reprimir algún tipo de manifestación en las diferencias en el crecimiento según tipo de estaca de tallo para taique. Probablemente, las ramas de donde fueron extraídas las estacas presentaban similares niveles nutricionales en relación a las reservas de carbohidratos (Hartmann y Kester 1991), lo que pudo haber influido en las respuestas similares de los dos tipos de estacas. Por otro lado, según Hartmann y Kester (1999), en especies que enraízan fácilmente, este factor es de poca importancia, cualquiera sea la posición en la rama. Esto concuerda con lo experimentado en este ensayo, puesto que taique no presentó dificultades para el enraizamiento.

Porcentajes de supervivencia y de enraizamiento de taique y tepa. Los porcentajes de sobrevivencia y enraizamiento de taique fueron relativamente altos, principalmente en los esquejes a los cuales se les aplicó hormona AIB. Sin embargo, cabe destacar que posiblemente la causa de que muchos de los esquejes no lograran enraizar y posteriormente murieran fue la humedad ambiental demasiado baja, en contraste con el sustrato, que por exceso de humedad puede haber presentado problemas de aireación, afectando la formación inicial de raíz. Esta situación se atribuye a que el sistema de riego no fue el más adecuado en respuesta a la dinámica hídrica de los esquejes, ya que no estuvo regulado por la humedad ambiental, factor de suma importancia para la propagación vegetativa mediante estacas, ya que antes de que éstas formen raíces son las hojas las que mantienen el balance hídrico dentro de los esquejes (Hartmann y Kester 1999).

Tepa es una especie que presenta ciertos problemas para la reproducción vegetativa, ya que el porcentaje más alto obtenido en este estudio fue un $33 \%$, valor que corresponde a la mayor concentración de hormona utilizada (4.000 $\mathrm{mg} \mathrm{L}^{-1}$ ). Este resultado valida lo descrito por Escobar ${ }^{2}$, quien también encontró dificultades en la propagación vegetativa de esta especie. Escobar logró este mismo porcentaje de enraizamiento (33\%), pero recién a los 10 meses de establecido el ensayo en cama fría y aplicando $2.000 \mathrm{mg} \mathrm{L}^{-1}$ de AIB. Lo anterior indica que tepa, en el presente ensayo, mostró una reacción favorable en el porcentaje de enraizamiento en menor tiempo de plantación, lo cual se atribuye a dos factores: La concentración utilizada que arrojó el mejor resultado en el presente ensayo fue mayor a la usada por Escobar y las condiciones para la propagación obedecen a las recomendaciones de Santelices (1990, 1991), quien asegura que los esquejes instalados en camas de arraigamiento a $20^{\circ} \mathrm{C}$ presentan una mejor supervivencia y desarrollo en diámetro y altura.
Sistemas radical y aéreo de taique. Esta especie mostró valores óptimos de desarrollo radical y aéreo para los tratamientos que utilizaron hormona, en comparación al tratamiento testigo, que es significativamente distinto al resto. Este resultado difiere con lo descrito por Awad (1993), quien tras propagar esta especie con distintas concentraciones de $\operatorname{AIB}(0,250,500,1.000,2.000,4.000)$ no encontró diferencias significativas en ninguno de los tratamientos utilizados en cuanto al desarrollo radical y aéreo de los esquejes. Esto se explicaría porque en el ensayo realizado por este autor se utilizó una cama de enraizamiento fría, en contraste a la temperatura de $22 \pm 2^{\circ} \mathrm{C}$ dada en este ensayo, lo cual demuestra una vez más el efecto positivo de la temperatura basal en el enraizamiento (Santelices 1990, 1991).

Por otro lado, las concentraciones 2.500 y $4.000 \mathrm{mg}$ $\mathrm{L}^{-1}$ de AIB lograron respuestas similares, por lo cual no se justificaría la aplicación de la mayor concentración, puesto que se estaría incurriendo en gastos innecesarios al aumentar la dosis.

Con respecto a las desviaciones estándar se destaca que éstas son altas, lo cual se atribuye a las distintas deficiencias estructurales que presenta el invernadero. Una de éstas, como ya fue mencionado, fue el sistema de riego, el cual no estuvo regulado por la humedad relativa del ambiente. Por otro lado, hubo sectores dentro de la cama de propagación que el riego no abarcó, por lo que se dio como solución a este problema el riego manual. Con esta solución no se obtuvieron buenos resultados durante la primavera, donde las temperaturas del ambiente comenzaron a subir y fue imprescindible un sistema de riego que regulara la humedad relativa, de manera de mantener ésta lo más cercana al $100 \%$. En consecuencia, bajó la temperatura del aire y hojas, disminuyendo el grado de transpiración. La falta de este sistema requirió mucha dedicación al riego, lo cual se tradujo en costo de oportunidad en términos de tiempo y un aumento en la mortalidad de los esquejes producto de la deshidratación de éstos.

Finalmente, el número muestral utilizado en este ensayo demostró no ser óptimo debido a que las desviaciones estándar fueron altas a muy altas, por lo cual se recomienda aumentar el número de estacas por tratamiento para no incurrir en mayores errores a la hora de evaluar los tratamientos.

Sistemas radical y aéreo de tepa. Esta especie presentó un desarrollo radical y aéreo muy limitado, siendo prácticamente los esquejes a los cuales se aplicó la concentración más alta (4.000 $\mathrm{mg} \mathrm{L}^{-1}$ de AIB) los que lograron emerger raíces y brotes. Además, la mayoría de los esquejes perdió sus hojas. Según Hechenleitner et al. (2005), la causal de esta situación pudiera ser que la estaca está estresada por falta de agua, por lo cual recomienda reducir el estrés aumentando la frecuencia de riego. Esta referencia confirma la situación experimentada 
en este ensayo, ya que el riego, al no estar regulado por la humedad ambiental, influyó negativamente en la obtención de mejores resultados, ya que disminuyó la supervivencia y el enraizamiento.

Por otro lado, se destaca que tepa presentó mayores dificultades para el enraizamiento y mayor sensibilidad a las condiciones de humedad del ambiente y sustrato que taique, lo cual se atribuyó a la autoecología de las especies. Taique es más plástica que tepa puesto que habita lugares muy húmedos (anegados) o muy secos en su hábitat natural, mientras que tepa habita en suelos húmedos, pero profundos y de buen drenaje (Donoso 2005). Estas características autoecológicas pudieron haber sido otro factor influyente en el mayor enraizamiento de taique y menor en tepa, puesto que el sustrato de propagación estuvo bastante húmedo a lo largo de todo el ensayo, lo que provocó en algunas estacas de tepa pudrición de la base y el follaje fue rápidamente infectado por hongos, síntoma no observable en taique.

Finalmente, según lo observado en terreno, tepa rebrota de tocón sin problemas cuando su hábitat natural es intervenido; sin embargo, su reproducción vegetativa bajo invernadero fue muy difícil, probablemente debido a que las condiciones ambientales no estuvieron totalmente controladas y/o la interacción de la hormona AIB con el enraizamiento no fue óptimo, ya que pese a que con la concentración $4.000 \mathrm{mg} \mathrm{L}^{-1}$ se obtuvo el mejor resultado, la producción media de brotes y raíces sigue siendo muy baja para la especie. Debido a esta situación, se recomienda mejorar las condiciones de propagación, ampliar el tiempo de estudio, experimentar el enraizamiento con concentraciones más altas de AIB (ya que no se ha observado toxicidad con las concentraciones usadas) o probar con otro tipo de hormona a fin de obtener mejores resultados para esta especie.

\section{CONCLUSIONES}

Taique no presentó diferencias significativas en el desarrollo radical ni aéreo según los distintos tipos de estaca utilizada (de la sección apical o subapical de la rama), de lo cual se infiere que este factor no influye en el enraizamiento. La capacidad de enraizamiento se vio beneficiada con la aplicación de hormona (AIB), presentando una relación positiva en el porcentaje de enraizamiento con la concentración de AIB.

La producción de raíces y brotes también presentó un incremento en sus valores medios a medida que aumentó la concentración de AIB; sin embargo, esta situación fue perceptible sólo para los tres primeros tratamientos (testigo, 1.000 y $2.500 \mathrm{mg} \mathrm{L}^{-1}$ ), puesto que con la concentración más alta $\left(4.000 \mathrm{mg} \mathrm{L}^{-1}\right)$, pese a no ser tóxica y mostrar resultados favorables, no presentó diferencias significativas con respecto a la concentración de $2.500 \mathrm{mg} \mathrm{L}{ }^{-1}$.
Tepa alcanza una alta supervivencia (85\%); sin embargo, su enraizamiento fue muy bajo, oscilando entre $6,7 \%$ para los esquejes sin hormona y un $33 \%$ para los esquejes tratados con $4.000 \mathrm{mg} \mathrm{L}^{-1}$ de AIB, lo cual demuestra una muy baja interacción de esta especie con la hormona utilizada y el enraizamiento.

\section{AGRADECIMIENTOS}

Se agradece al proyecto FIA-PI-C-2005-1-A-114 (Mejoramiento de especies nativas para follaje ornamental y producción bajo condiciones controladas) y a Southgreen por haber financiado y permitido trabajar en esta empresa.

\section{REFERENCIAS}

Awad G. 1993. Propagación vegetativa de seis especies vegetales nativas con posibilidades ornamentales. Tesis Ing. Agrónomo. Valdivia, Chile. Universidad Austral de Chile, Fac. de Cs. Agrarias. 66 p.

Donoso C, A Cabello. 1978. Antecedentes fenológicos y de germinación de especies leñosas chilenas. Ciencias Forestales 1(2): 31-34.

Donoso C. 1983. Árboles nativos de Chile. Guía de reconocimiento. Valdivia, Chile. Alborada. 116 p.

Donoso C. 2005. Árboles nativos de Chile. Guía de reconocimiento. $11^{\mathrm{a}}$ ed. Valdivia, Chile. Marisa Cuneo Ediciones. 136 p.

Donoso C. 2006. Las especies arbóreas de los bosques templados de Chile y Argentina. Autoecología. Valdivia, Chile. Marisa Cuneo Ediciones. 678 p.

FIA (Fondo para la Innovación Agraria, CL). 2005. Mejoramiento de especies nativas para follaje ornamental y producción bajo condiciones controladas. Código: FIA-PI-C-20051-A-114.

Hartmann T, D Kester. 1991. Propagación de plantas: principios y prácticas. México DF, México. Continental. 810 p.

Hartmann T, D Kester. 1999. Propagación de plantas. Principios y prácticas. Séptima reimpresión. México DF, México. Continental. $757 \mathrm{p}$.

Hechenleitner P, M Gardner, P Thomas, C Echeverría, B Escobar, P Brownless, A Martínez. 2005. Plantas amenazadas del centro-sur de Chile. Distribución, conservación y propagación. Valdivia, Chile. Universidad Austral de Chile y Real Jardín Botánico de Edimburgo. 188 p.

Palma J. 2003. Caracterización de las unidades de recolección del follaje nativo ornamental de Lomatia ferruginea (Cav.) R. Br., Gevuina avellana Mol., Lycopodium paniculatum A. N. Desv. y Dendroligotrichum dendroides (Brid. ex Hedw) Broth., en cuatro comunidades huilliches de San Juan de la Costa, X Región. Tesis Ing. Forestal. Temuco, Chile. Universidad de la Frontera, Fac. de Cs. Agropecuarias y Forestales. 84 p.

Riedemann P, G Aldunate. 2003. Flora nativa de valor ornamental. Chile, zona sur. Identificación y propagación. Santiago, Chile. Andrés Bello. 516 p. 
Santelices R. 1990. Propagación vegetativa de tepa (Laureliopsis philippiana) a partir de estacas. Ciencia e Investigación Forestal 4(1): 61-68.

Santelices R. 1991. Propagación vegetativa de tepa (Laureliopsis philippiana), lingue (Persea lingue) y mañío (Podocarpus saligna) a partir de estacas. Ciencia e Investigación Forestal 5 (2): 195-202.
Tacón A, J Palma, U Fernández, O Ortega. 2005. El mercado de los productos forestales no madereros y la conservación de los bosques del sur de Chile y Argentina. Red de productos forestales no madereros de Chile. Valdivia, Chile. WWF Chile. 95 p.

Recibido: 29.08 .07

Aceptado: 28.03.08 